\title{
Decreased carotid and vertebral arterial blood-flow velocity in response to orthostatic unload in patients with severe aortic stenosis
}

\author{
Paweł Kleczyński, Paweł Petkow Dimitrow, Artur Dziewierz, \\ Andrzej Surdacki, Dariusz Dudek \\ Institute of Cardiology, Krakow, Poland
}

\begin{abstract}
Background: Responses of cerebral blood flow to the postural unloading maneuver in aortic stenosis (AS) have not been described so far. Our aim was to assess effects of orthostatic stress test on changes of carotid and vertebral artery blood flow and transaortic gradients.

Methods: From consecutive 101 AS patients we selected 50 patients with severe isolated AS. Maximal and mean transaortic pressure gradients, as well as peak systolic blood-flow velocity (PSV) and end-diastolic velocity (EDV) in the common carotid artery, internal carotid artery and vertebral artery on both sides were measured by duplex ultrasound in the supine position and at 1-2 min after the assumption of the sitting position in patients with AS, and in standing position in healthy controls.

Results: The orthostatic stress test induced significant decrease of carotid and vertebral arterial flow velocities in AS patients. Transaortic pressure gradients also dropped while the patients were sitting $(p<0.001)$. A history of syncope/presyncope was not associated with a significantly lower PSV and EDV in carotid and vertebral arteries in the upright position. In healthy controls, the velocities in carotid and vertebral arterial flow have been unchanged after maneuver reducing preload.

Conclusions: In AS patients, decrease of carotid and vertebral arterial flow velocities and transaortic gradients in the sitting position were observed. Orthostatic test position does not appear to be associated with a history of syncope/presyncope in patients with severe isolated $A S$, despite a simultaneous drop of transvalvular pressure gradient. (Cardiol J 2016; 23, 4: 393-401)
\end{abstract}

Key words: aortic stenosis, carotid Doppler ultrasound, orthostatic stress

\section{Introduction}

Aortic stenosis (AS) is the most frequent type of valvular heart disease all over the world and affects mostly adults of advanced age (2-7\% of the population $>65$ years) with its primarily calcific form $[1,2]$. Several studies have reported the importance of upright positioning during ex- ercise in the detection of provocable (latent) or labile left ventricular (LV) outflow tract gradients in hypertrophic cardiomyopathy [3-12]. Also, passive orthostatic tests (without exercise) are helpful in diagnostic evaluation in other diseases predisposing to LV outflow tract gradients $[8,12]$. Previously, the orthostatic response of valvular gradients in AS has been performed and gener-

Address for correspondence: Paweł Kleczyński, MD, PhD, Institute of Cardiology, ul. Kopernika 17, 31-501 Kraków, Poland, tel: +48 1242471 81, fax: +48 1242471 84, e-mail: kleczu@interia.pl

Received: 31.01.2016 Accepted: 09.05.2016 
ated a decrease in transvalvular gradient in the majority of patients with AS [13]. Carotid duplex ultrasound is an accurate noninvasive technique to estimate velocity spectral analysis in extracranial carotid and vertebral arteries with measurements of peak systolic velocity (PSV) and end diastolic velocity (EDV). To the best of our knowledge, this is the first study to evaluate carotid flow pattern assessed by duplex ultrasound in patients with severe AS, additionally in non-supine position. The aim of this study was to compare the effects of the orthostatic stress test on carotid and vertebral artery blood flow and transaortic gradients according to a history of syncope or presyncope in severe AS. Additionally, AS group has been compared with healthy controls.

\section{Methods}

The present study included 101 consecutive patients who underwent comprehensive echocardiography and carotid duplex ultrasound in our department. The inclusion criteria were as follows: severe "isolated" AS, defined as an aortic valve area (AVA) $<1.0 \mathrm{~cm}^{2}$; preserved LV ejection fraction ( $>50 \%)$; no significant atherosclerosis in carotid and vertebral arteries; and sinus rhythm. To omit potentially disturbing factors, 42 patients were excluded from the study due to detected atherosclerosis in carotid/vertebral arteries. Additional exclusion criteria were non-severe AS and atrial flutter/fibrillation. From technical point of view, the next exclusion from the study was a problem with suboptimal Doppler signal during orthostatic stress. Patients with more than mild concomitant mitral valve dysfunction were also excluded, as well as patients with concomitant more than mild aortic insufficiency. The apical window did not allow estimating the transvalvular gradients in 9 patients. The following clinical data were collected: age, gender, hypercholesterolemia, diabetes mellitus, and previous evidence of coronary artery disease (presence of $>50 \%$ coronary artery stenosis on angiography, previous revascularization or previous myocardial infarction). The relevant institutional Review Board approved the protocol. Standard echocardiography was performed in the supine position using Vivid 7 (General Electric, Fairfield, USA). We obtained the M-mode and 2-dimensional echocardiograms for each patient which was followed by a pulsed and continuous-wave Doppler ultrasound. We used conventional techniques to measure the echocardiographic parameters. During first part of orthostatic test, the patient sat for
1-2 min with their left hand on their head and the gradient assessment from the apical window was performed. The ultrasound transducer $(4-10 \mathrm{MHz}$ linear-array transducer) was used to perform carotid duplex ultrasound routinely in supine position with PSV and EDV assessment in common carotid, internal carotid, and vertebral arteries. In the second part of the examination, during the orthostatic test, the patient sat for 1-2 min and carotid duplex ultrasound was performed again with velocity measurements, respectively. Heart rate was assessed at baseline and after 1-2 min of orthostatic stress. Additionally, we assessed the diameter of left and right common carotid artery (LCCA and RCCA, respectively), spectral analysis of flow pattern with time-averaged maximum velocity (TAMAX $[\mathrm{cm} / \mathrm{s}])$, time-averaged mean velocity (TAMEAN $[\mathrm{cm} / \mathrm{s}]$ ) and flow volume $[\mathrm{mL} / \mathrm{min}]$ at baseline and in upright position. Control group in our study consisted of 40 healthy subjects, strictly selected also with same criteria as patients from the study group. Echocardiographic assessment and Doppler ultrasound was performed in supine position, as previously described. The orthostatic test was more meaningful because we introduced upright position for assessment of blood flow in carotid arteries. During orthostatic stress, subjects were examined in a standing position, after 1-2 min of orthostatic stress. Each echocardiographic and Doppler ultrasound parameter was assessed repeatedly 3 times and mean value was taken into analysis.

\section{Statistical analysis}

Continuous variables were presented as medians (interquartile $[\mathrm{IQR}]$ ranges) or mean \pm standard deviations. Categorical variables were expressed as numbers (percentages). Differences between patients with and without syncope/presyncope were tested using $\chi^{2}$ test and the Fisher's exact test for dichotomous variables and the Mann-Whitney U test for continuous variables. Differences between echocardiographic/duplex ultrasound parameters assessed in supine and sitting/upright position were assessed using Wilcoxon signed-rank test. Comparison between measured parameters of AS patients with control group were assessed using paired samples t-test. Independent predictors of syncope/presyncope were identified using multivariate logistic regression analysis. Forward selection in logistic regression with the probability value for covariates to enter the model were set at 0.05 . All reported clinical, echocardiographic, and duplex ultrasound data were tested 
Table 1. Baseline clinical characteristics of the patients.

\begin{tabular}{lc}
\hline Variable & $\mathbf{N}=50$ \\
\hline Male gender & $23(46.0 \%)$ \\
Age [years] & $70.0(65.8,74.3) ;$ \\
& $70.2 \pm 6.0$ \\
Diabetes mellitus & $11(22.0 \%)$ \\
Hypercholesterolemia & $49(98.0 \%)$ \\
Coronary artery disease & $46(92.0 \%)$ \\
Peripheral arterial disease & $6(12.0 \%)$ \\
Chronic obstructive & $4(8.0 \%)$ \\
pulmonary disease & $7(14.0 \%)$ \\
Chronic kidney disease & $5(10.0 \%)$ \\
Syncope & $24(48.0 \%)$ \\
Presyncope &
\end{tabular}

as possible covariates. Relative risks of syncope/ presyncope were expressed as odds ratios (OR) with $95 \%$ confidence intervals (CI). All tests were 2 -tailed, and a $\mathrm{p}$ value of $<0.05$ was considered statistically significant. All statistical analyses were performed using SPSS software, version 15.0 (SPSS Inc., Chicago, Illinois).

\section{Results}

Table 1 reports demographic and clinical variables, while Table 2 depicts echocardiographic characteristics of the AS study population. Fifty patients were included in the study with median age of 70 years among who 23 were males. Median AVA was 0.9 [IQR $0.8-0.9] \mathrm{cm}^{2}$, median transaortic maximal/mean gradient was $99 / 48.5 \mathrm{~mm} \mathrm{Hg}$ in supine position, and $90 / 42 \mathrm{~mm} \mathrm{Hg}$ in upright position ( $\mathrm{p}<0.001$ ). Median heart rate in AS patients was 77.0 (IQR 67.0-81.0) at baseline and 76.0 (IQR 67.0-82.0) in upright position $(\mathrm{p}=0.83)$, and 80.0 (IQR 71.0-85.0) at baseline and 81.0 (IQR $72.0-86.0)$ while standing in the control group $(\mathrm{p}=$
0.85). Diameter of LCCA and RCCA was $0.69 \mathrm{~cm}$ (IQR 0.65-0.71). Median PSV and EDV values and TAMAX, TAMEAN, and flow volume for LCCA and RCCA are shown in Table 3. The orthostatic stress test (sitting position) induced significant decreases of carotid and vertebral arterial flow velocities and also significant changes in flow volume in LCCA and RCCA. Transaortic pressure gradients also dropped on standing position (maximal gradient: from 99 [IQR 93-103.3] to 90 [IQR 86.8-93] mm $\mathrm{Hg}, \mathrm{p}<0.001$; mean gradient: from 48.5 [IQR 45-52.3] to 42 [IQR 41-45.5] mm Hg, $\mathrm{p}<0.001$ ). A history of syncope/presyncope was not associated with a significantly lower PSV and EDV in carotid and vertebral arteries in the sitting position.

Additionally, a subanalysis comparing patients with and without syncope/presyncope was performed (Table 4). Patients with syncope/presyncope were older $(p=0.001)$, more often had a history of peripheral artery disease $(\mathrm{p}=0.003)$ and chronic obstructive pulmonary disease $(\mathrm{p}=0.026)$, chronic kidney disease $(p=0.001)$, smaller AVA $(\mathrm{p}=0.016)$ and higher maximal $(\mathrm{p}=0.016)$ and mean $(\mathrm{p}=0.007)$ transaortic gradients. In the multivariate logistic regression analysis, the only independent predictor of syncope/presyncope was patients' age (OR 1.68 per 1 year, 95\% CI: 1.24, 2.27; $\mathrm{p}=0.001)$. Similarly, patients' age was an independent predictor of syncope (OR 2.22 per 1 year, 95\% CI: 1.26, 3.92; p = 0.006), and both patients' age (OR 1.81 per 1 year, 95\% CI: 1.25 , $2.63 ; \mathrm{p}=0.002]$ and AVA (OR 0.003 per $1 \mathrm{~cm}^{2} ; 95 \%$ CI: $0.000-0.498 ; p=0.026$ ] were independently associated with prescyncope.

We also performed a subanalysis of orthostatic stress in patients with aortic stenosis and healthy subjects. Subjects from the control group were younger than patients with AS (45.0 [IQR 30.5-58.5], $\mathrm{p}<0.001)$. Carotid Doppler ultrasound data of the controlled group are presented in Table 3.

Comparison of carotid Doppler ultrasound measurements is shown in Table 5 and Figure 1.

Table 2. Echocardiographic data of patients with aortic stenosis.

\begin{tabular}{lccc}
\hline Variable & Supine & Upright & P \\
\hline TG maximal $[\mathrm{mm} \mathrm{Hg}]$ & $99.0(93.0 ; 103.3)$ & $90.0(86.8 ; 93.0)$ & $<0.001$ \\
TG mean $[\mathrm{mm} \mathrm{Hg}]$ & $48.5(45.0 ; 52.3)$ & $42.0(41.0 ; 45.5)$ & $<0.001$ \\
LVEF $[\%]$ & $60.0(58.8 ; 65.0)$ & - & - \\
Aortic valve area $\left[\mathrm{cm}^{2}\right]$ & $0.9(0.8 ; 1.0)$ & - & - \\
\hline
\end{tabular}

TG — transaortic gradient; LVEF — left ventricular ejection fraction 
Table 3. Carotid duplex ultrasound data.

\begin{tabular}{|c|c|c|c|}
\hline Variable & Supine & Sitting & $\mathbf{P}$ \\
\hline \multicolumn{4}{|l|}{ Patients with aortic stenosis } \\
\hline PSV LCCA [cm/s] & $95.5(89.0 ; 101.3)$ & $86.5(82.0 ; 90.0)$ & $<0.001$ \\
\hline EDV LCCA $[\mathrm{cm} / \mathrm{s}]$ & $25.0(22.0 ; 27.0)$ & $20.0(19.0 ; 22.0)$ & $<0.001$ \\
\hline TAMAX LCCA $[\mathrm{cm} / \mathrm{s}]$ & $48.5(44.2-50.0)$ & $45.2(43.2-46.7)$ & $<0.001$ \\
\hline TAMEAN LCCA $[\mathrm{cm} / \mathrm{s}]$ & $24.6(22.9-25.2)$ & $22.4(21.5-23.7)$ & $<0.001$ \\
\hline Flow volume LCCA [mL/min] & $698.9(632.3-733.4)$ & $676.7(615.8-703.3)$ & $<0.001$ \\
\hline PSV LICA [cm/s] & $89.0(85.8 ; 95.3)$ & $83.5(80.0 ; 87.0)$ & $<0.001$ \\
\hline EDV LICA [cm/s] & $21.0(20.0 ; 23.0)$ & $18.0(15.0 ; 19.0)$ & $<0.001$ \\
\hline PSV LVA [cm/s] & $42.5(38.0 ; 47.0)$ & $38.0(36.0 ; 40.0)$ & $<0.001$ \\
\hline EDV LVA [cm/s] & $12.0(10.8 ; 14.0)$ & $9.0(8.0 ; 11.0)$ & $<0.001$ \\
\hline PSV RCCA [cm/s] & $92.5(85.0 ; 100.0)$ & $85.0(80.8 ; 91.0)$ & $<0.001$ \\
\hline EDV RCCA $[\mathrm{cm} / \mathrm{s}]$ & $23.5(21.0 ; 26.0)$ & $20.0(19.0 ; 21.0)$ & $<0.001$ \\
\hline TAMAX RCCA [cm/s] & $48.0(44.2-51.0)$ & $45.8(42.0-48.0$ & $<0.001$ \\
\hline TAMEAN RCCA [cm/s] & $24.0(22.9-26.0)$ & $22.1(21.3-23.8)$ & $<0.001$ \\
\hline Flow volume RCCA [mL/min] & $698.4(632.8-732.9)$ & $675.2(615.2-703.7)$ & $<0.001$ \\
\hline PSV RICA $[\mathrm{cm} / \mathrm{s}]$ & $88.0(83.8 ; 93.0)$ & $80.0(75.8 ; 85.0)$ & $<0.001$ \\
\hline EDV RICA $[\mathrm{cm} / \mathrm{s}]$ & $20.0(19.0 ; 22.0)$ & $19.0(15.0 ; 20.0)$ & $<0.001$ \\
\hline PSV RVA $[\mathrm{cm} / \mathrm{s}]$ & $44.0(37.8 ; 46.0)$ & $37.0(36.0 ; 39.0)$ & $<0.001$ \\
\hline EDV RVA $[\mathrm{cm} / \mathrm{s}]$ & $12.5(11.0 ; 14.0)$ & $9.0(8.0 ; 11.0)$ & $<0.001$ \\
\hline \multicolumn{4}{|l|}{ Control group } \\
\hline PSV LCCA [cm/s] & $125.0(120.0 ; 130.5)$ & $124.0(120.0 ; 130.0)$ & 0.004 \\
\hline EDV LCCA [cm/s] & $23.0(19.5 ; 24.5)$ & $22.0(19.0 ; 24.0)$ & 0.001 \\
\hline PSV LICA [cm/s] & $123.0(117.5 ; 126.0)$ & $123.0(117.5 ; 129.0)$ & 0.46 \\
\hline EDV LICA [cm/s] & $20.0(17.0 ; 21.5)$ & $20.0(17.0 ; 21.0)$ & $<0.001$ \\
\hline PSV LVA $[\mathrm{cm} / \mathrm{s}]$ & $45.0(43.0 ; 46.5)$ & $44.0(43.0 ; 46.0)$ & 0.50 \\
\hline EDV LVA $[\mathrm{cm} / \mathrm{s}]$ & $9.0(8.0 ; 10.0)$ & $9.0(8.0 ; 10.0)$ & 0.98 \\
\hline PSV RCCA [cm/s] & $125.0(120.0 ; 130.5)$ & $124.0(120.0 ; 130.0)$ & 0.006 \\
\hline EDV RCCA $[\mathrm{cm} / \mathrm{s}]$ & $23.0(19.5 ; 25.0)$ & $22.0(19.0 ; 24.0)$ & 0.010 \\
\hline PSV RICA [cm/s] & $123.0(117.0 ; 126.0)$ & $123.0(118.0 ; 128.0)$ & 0.82 \\
\hline EDV RICA $[\mathrm{cm} / \mathrm{s}]$ & $20.0(17.0 ; 21.0)$ & $20.0(17.0 ; 21.0)$ & 0.030 \\
\hline PSV RVA $[\mathrm{cm} / \mathrm{s}]$ & $45.0(43.0 ; 46.0)$ & $44.0(43.0 ; 46.0)$ & 0.35 \\
\hline EDV RVA $[\mathrm{cm} / \mathrm{s}]$ & $9.0(8.0 ; 11.0)$ & $9.0(7.0 ; 10.5)$ & 0.005 \\
\hline
\end{tabular}

EDV — end diastolic velocity; PSV — peak systolic velocity; LCCA — left common carotid artery; LICA — left internal carotid artery; LVA - left vertebral artery; RCCA — right common carotid artery; RICA — right internal carotid artery; RVA — right vertebral artery; TAMAX - time-averaged maximum velocity; TAMEAN — time-averaged mean velocity

Despite the difference in PSV and EDV values in supine position, we found statistical significance in deltas after orthostatic stress. Despite less reducing LV load (sitting vs. erect position), the drop is observed in patients with AS. Probably, this could be even more emphasized if one examined those patients in standing and not sitting position. In Figure 2, we present a sample of Doppler ultrasound assessment in a patient with AS and a healthy subject.

\section{Discussion}

An increasing prevalence of patients with valvular heart disease requires a dedicated management approach [13]. A patient with severe AS should undergo a Doppler ultrasound examination of carotid and vertebral arteries only in limited conditions before surgical aortic valve replacement or transcatheter aortic valve implantation. The evaluation of valve disorder only at resting condi- 
Table 4. Comparison of patients with and without syncope/presyncope.

\begin{tabular}{|c|c|c|c|c|c|}
\hline \multirow[t]{2}{*}{ Variable } & & \multicolumn{2}{|c|}{ Presyncope/Syncope } & \multirow[t]{2}{*}{$\mathbf{P}$} & \multirow[t]{2}{*}{ OR (95\% CI) } \\
\hline & & $\begin{array}{c}\text { No } \\
(n=29)\end{array}$ & $\begin{array}{c}\text { Yes } \\
(n=21)\end{array}$ & & \\
\hline Male gender & & $51.7 \%$ & $38.1 \%$ & 0.34 & $0.57(0.18 ; 1.80)$ \\
\hline Age [year] & & $66.7 \pm 4.4$ & $74.9 \pm 4.4$ & 0.001 & $1.68(1.24 ; 2.27)$ \\
\hline Diabetes mellitus & & $20.7 \%$ & $23.8 \%$ & 0.79 & $1.20(0.31 ; 4.61)$ \\
\hline Hypercholesterolemia & & $96.6 \%$ & $100.0 \%$ & 1.00 & - \\
\hline Coronary artery disease & & $86.2 \%$ & $100.0 \%$ & 1.00 & - \\
\hline Peripheral arterial disease & & $0.0 \%$ & $28.6 \%$ & 0.003 & - \\
\hline Chronic obstructive pulmonary disease & & $0.0 \%$ & $19.0 \%$ & 0.026 & - \\
\hline Chronic kidney disease & & $0.0 \%$ & $33.3 \%$ & 0.001 & - \\
\hline Left ventricular ejection fraction [\%] & & $62.1 \pm 3.8$ & $58.8 \pm 3.9$ & 0.010 & $0.80(0.68 ; 0.95)$ \\
\hline Aortic valve area $\left[\mathrm{cm}^{2}\right]$ & & $0.9 \pm 0.2$ & $0.7 \pm 0.1$ & 0.016 & $0.004(0.00 ; 0.35)$ \\
\hline \multirow{3}{*}{$\mathrm{TG} \max [\mathrm{mm} \mathrm{Hg}]$} & Supine & $96.8 \pm 7.5$ & $104.5 \pm 11.0$ & 0.016 & $1.11(1.02 ; 1.21)$ \\
\hline & Sitting & $88.5 \pm 7.0$ & $95.7 \pm 9.3$ & 0.016 & $1.15(1.03 ; 1.29)$ \\
\hline & Delta & $-8.2 \pm 3.6$ & $-8.8 \pm 3.6$ & 0.57 & $0.96(0.81 ; 1.12)$ \\
\hline \multirow[t]{3}{*}{ TG mean [mm Hg] } & Supine & $47.0 \pm 4.7$ & $51.8 \pm 5.8$ & 0.007 & $1.19(1.05 ; 1.36)$ \\
\hline & Sitting & $41.9 \pm 3.4$ & $45.7 \pm 4.7$ & 0.007 & $1.28(1.07 ; 1.54)$ \\
\hline & Delta & $-5.1 \pm 3.0$ & $-6.1 \pm 2.2$ & 0.25 & $0.88(0.71 ; 1.09)$ \\
\hline \multirow[t]{3}{*}{ PSV LCCA $[\mathrm{cm} / \mathrm{s}]$} & Supine & $94.6 \pm 8.9$ & $95.0 \pm 8.6$ & 0.86 & $1.00(0.94 ; 1.07)$ \\
\hline & Sitting & $84.6 \pm 6.0$ & $87.4 \pm 7.2$ & 0.14 & $1.08(0.98 ; 1.19)$ \\
\hline & Delta & $-10.0 \pm 7.8$ & $-7.6 \pm 3.1$ & 0.20 & $1.07(0.97 ; 1.18)$ \\
\hline \multirow[t]{3}{*}{ EDV LCCA [cm/s] } & Supine & $25.2 \pm 2.3$ & $23.9 \pm 3.1$ & 0.09 & $0.82(0.66 ; 1.03)$ \\
\hline & Sitting & $21.0 \pm 1.9$ & $20.0 \pm 1.7$ & 0.021 & $0.67(0.47 ; 0.94)$ \\
\hline & Delta & $-4.3 \pm 2.8$ & $-4.2 \pm 2.7$ & 0.96 & $1.00(0.82 ; 1.24)$ \\
\hline \multirow[t]{3}{*}{ PSV LICA [cm/s] } & Supine & $88.3 \pm 6.9$ & $91.0 \pm 8.5$ & 0.23 & $1.05(0.97 ; 1.14)$ \\
\hline & Sitting & $81.8 \pm 5.8$ & $83.4 \pm 6.7$ & 0.38 & $1.05(0.95 ; 1.16)$ \\
\hline & Delta & $-6.5 \pm 4.6$ & $-7.6 \pm 3.7$ & 0.35 & $0.94(0.81 ; 1.08)$ \\
\hline \multirow[t]{3}{*}{ EDV LICA $[\mathrm{cm} / \mathrm{s}]$} & Supine & $21.1 \pm 2.2$ & $21.2 \pm 2.2$ & 0.79 & $1.04(0.80 ; 1.35)$ \\
\hline & Sitting & $17.7 \pm 2.4$ & $16.9 \pm 2.2$ & 0.22 & $0.85(0.67 ; 1.10)$ \\
\hline & Delta & $-3.4 \pm 3.6$ & $-4.4 \pm 3.1$ & 0.31 & $0.92(0.78 ; 1.08)$ \\
\hline \multirow[t]{3}{*}{ PSV LVA $[\mathrm{cm} / \mathrm{s}]$} & Supine & $40.8 \pm 6.7$ & $43.0 \pm 7.9$ & 0.28 & $1.05(0.96 ; 1.14)$ \\
\hline & Sitting & $36.9 \pm 5.0$ & $36.9 \pm 4.7$ & 1.00 & $1.00(0.89 ; 1.13)$ \\
\hline & Delta & $-3.9 \pm 5.0$ & $-6.1 \pm 5.7$ & 0.15 & $0.92(0.83 ; 1.03)$ \\
\hline \multirow[t]{3}{*}{ EDV LVA [cm/s] } & Supine & $11.6 \pm 2.9$ & $12.6 \pm 2.5$ & 0.22 & $1.15(0.92 ; 1.42)$ \\
\hline & Sitting & $9.9 \pm 2.4$ & $9.6 \pm 1.9$ & 0.61 & $0.93(0.71 ; 1.22)$ \\
\hline & Delta & $-1.7 \pm 3.2$ & $-3.0 \pm 2.8$ & 0.15 & $0.87(0.72 ; 1.05)$ \\
\hline \multirow[t]{3}{*}{ PSV RCCA [cm/s] } & Supine & $89.9 \pm 8.0$ & $94.5 \pm 9.0$ & 0.07 & $1.07(1.00 ; 1.16)$ \\
\hline & Sitting & $82.8 \pm 6.8$ & $87.9 \pm 6.9$ & 0.020 & $1.13(1.02 ; 1.26)$ \\
\hline & Delta & $-7.0 \pm 6.7$ & $-6.6 \pm 6.6$ & 0.81 & $1.01(0.93 ; 1.10)$ \\
\hline \multirow[t]{3}{*}{ EDV RCCA [cm/s] } & Supine & $23.3 \pm 2.6$ & $24.0 \pm 3.1$ & 0.45 & $1.08(0.88 ; 1.33)$ \\
\hline & Sitting & $20.2 \pm 1.7$ & $19.5 \pm 1.8$ & 0.20 & $0.80(0.57 ; 1.13)$ \\
\hline & Delta & $-3.2 \pm 2.9$ & $-4.4 \pm 2.2$ & 0.10 & $0.82(0.65 ; 1.04)$ \\
\hline \multirow[t]{3}{*}{ PSV RICA [cm/s] } & Supine & $87.2 \pm 6.4$ & $89.8 \pm 8.5$ & 0.23 & $1.05(0.97 ; 1.15)$ \\
\hline & Sitting & $78.3 \pm 6.8$ & $82.5 \pm 6.3$ & 0.039 & $1.11(1.00 ; 1.23)$ \\
\hline & Delta & $-8.9 \pm 5.3$ & $-7.3 \pm 3.4$ & 0.28 & $1.08(0.95 ; 1.24)$ \\
\hline \multirow[t]{3}{*}{ EDV RICA $[\mathrm{cm} / \mathrm{s}]$} & Supine & $19.8 \pm 2.4$ & $20.8 \pm 2.6$ & 0.20 & $1.17(0.92 ; 1.47)$ \\
\hline & Sitting & $18.1 \pm 2.5$ & $16.7 \pm 3.5$ & 0.11 & $0.85(0.70 ; 1.04)$ \\
\hline & Delta & $-1.7 \pm 3.8$ & $-4.1 \pm 5.3$ & 0.08 & $0.89(0.78 ; 1.01)$ \\
\hline \multirow[t]{3}{*}{ PSV RVA [cm/s] } & Supine & $40.7 \pm 6.4$ & $42.6 \pm 7.9$ & 0.33 & $1.04(0.96 ; 1.14)$ \\
\hline & Sitting & $37.2 \pm 3.4$ & $36.5 \pm 5.5$ & 0.56 & $0.96(0.85 ; 1.10)$ \\
\hline & Delta & $-3.5 \pm 5.8$ & $-6.1 \pm 5.4$ & 0.11 & $0.92(0.82 ; 1.02)$ \\
\hline \multirow[t]{3}{*}{ EDV RVA $[\mathrm{cm} / \mathrm{s}]$} & Supine & $12.6 \pm 2.3$ & $12.9 \pm 1.9$ & 0.61 & $1.07(0.82 ; 1.40)$ \\
\hline & Sitting & $9.6 \pm 2.4$ & $10.1 \pm 2.6$ & 0.40 & $1.11(0.88 ; 1.40)$ \\
\hline & Delta & $-3.0 \pm 2.6$ & $-2.7 \pm 3.2$ & 0.72 & $1.04(0.85 ; 1.27)$ \\
\hline
\end{tabular}

EDV — end diastolic velocity; PSV — peak systolic velocity; LCCA — left common carotid artery; LICA — left internal carotid artery;

LVA - left vertebral artery; RCCA - right common carotid artery; RICA — right internal carotid artery; RVA — right vertebral artery;

TG - transaortic gradient; OR — odds ratio; $\mathrm{Cl}$ - confidence interval 
Table 5. Comparison of carotid Doppler ultrasound parameters of aortic stenosis (AS) patients with control group in terms of orthostatic stress.

\begin{tabular}{|c|c|c|c|c|}
\hline Variable & & AS patients $(n=50)$ & Control group $(n=40)$ & $\mathbf{P}$ \\
\hline \multirow[t]{3}{*}{ PSV LCCA [cm/s] } & Supine & $95.5(89.0 ; 101.3)$ & $125.0(120.0 ; 130.5)$ & $<0.001$ \\
\hline & Sitting/upright & $86.5(82.0 ; 90.0)$ & $124.0(120.0 ; 130.0)$ & $<0.001$ \\
\hline & Delta & $-7.0(-11.3 ;-4.0)$ & $-1.0(-2.0 ; 1.0)$ & $<0.001$ \\
\hline \multirow[t]{3}{*}{ EDV LCCA $[\mathrm{cm} / \mathrm{s}]$} & Supine & $25.0(22.0 ; 27.0)$ & $23.0(19.5 ; 24.5)$ & $<0.001$ \\
\hline & Sitting/upright & $20.0(19.0 ; 22.0)$ & $22.0(19.0 ; 24.0)$ & 0.05 \\
\hline & Delta & $-4.0(-6.0 ;-2.0)$ & $-1.0(-1.0 ;-0.0)$ & $<0.001$ \\
\hline \multirow[t]{3}{*}{ PSV LICA [cm/s] } & Supine & $89.0(85.8 ; 95.3)$ & $123.0(117.5 ; 126.0)$ & $<0.001$ \\
\hline & Sitting/upright & $83.5(80.0 ; 87.0)$ & $123.0(117.5 ; 129.0)$ & $<0.001$ \\
\hline & Delta & $-7.0(-10.0 ;-4.8)$ & $0.0(-1.0 ; 1.0)$ & $<0.001$ \\
\hline \multirow[t]{3}{*}{ EDV LICA [cm/s] } & Supine & $21.0(20.0 ; 23.0)$ & $20.0(17.0 ; 21.5)$ & 0.011 \\
\hline & Sitting/upright & $18.0(15.0 ; 19.0)$ & $20.0(17.0 ; 21.0)$ & 0.002 \\
\hline & Delta & $-3.0(-7.0 ;-1.0)$ & $0.0(-1.0 ; 0.0)$ & $<0.001$ \\
\hline \multirow[t]{3}{*}{ PSV LVA [cm/s] } & Supine & $42.5(38.0 ; 47.0)$ & $45.0(43.0 ; 46.5)$ & 0.029 \\
\hline & Sitting/upright & $38.0(36.0 ; 40.0)$ & $44.0(43.0 ; 46.0)$ & $<0.001$ \\
\hline & Delta & $-2.0(-10.0 ;-1.0)$ & $0.0(-1.0 ; 1.0)$ & $<0.001$ \\
\hline \multirow{3}{*}{ EDV LVA $[\mathrm{cm} / \mathrm{s}]$} & Supine & $12.0(10.8 ; 14.0)$ & $9.0(8.0 ; 10.0)$ & $<0.001$ \\
\hline & Sitting/upright & $9.0(8.0 ; 11.0)$ & $9.0(8.0 ; 10.0)$ & 0.12 \\
\hline & Delta & $-1.0(-5.0 ; 0.0)$ & $0.0(0.0 ; 0.0)$ & $<0.001$ \\
\hline \multirow[t]{3}{*}{ PSV RCCA $[\mathrm{cm} / \mathrm{s}]$} & Supine & $92.5(85.0 ; 100.0)$ & $125.0(120.0 ; 130.5)$ & $<0.001$ \\
\hline & Sitting/upright & $85.0(80.8 ; 91.0)$ & $124.0(120.0 ; 130.0)$ & $<0.001$ \\
\hline & Delta & $-6.0(-10.0 ;-2.8)$ & $-1.0(-1.5 ; 1.0)$ & $<0.001$ \\
\hline \multirow{3}{*}{ EDV RCCA $[\mathrm{cm} / \mathrm{s}]$} & Supine & $23.5(21.0 ; 26.0)$ & $23.0(19.5 ; 25.0)$ & 0.06 \\
\hline & Sitting/upright & $20.0(19.0 ; 21.0)$ & $22.0(19.0 ; 24.0)$ & 0.002 \\
\hline & Delta & $-4.0(-6.0 ;-2.0)$ & $-1.0(-1.0 ; 0.0)$ & $<0.001$ \\
\hline \multirow[t]{3}{*}{ PSV RICA [cm/s] } & Supine & $88.0(83.8 ; 93.0)$ & $123.0(117.0 ; 126.0)$ & $<0.001$ \\
\hline & Sitting/upright & $80.0(75.8 ; 85.0)$ & $123.0(118.0 ; 128.0)$ & $<0.001$ \\
\hline & Delta & $-8.0(-10.3 ;-5.8)$ & $0.0(-1.0 ; 1.0)$ & $<0.001$ \\
\hline \multirow[t]{3}{*}{ EDV RICA [cm/s] } & Supine & $20.0(19.0 ; 22.0)$ & $20.0(17.0 ; 21.0)$ & 0.35 \\
\hline & Sitting/upright & $19.0(15.0 ; 20.0)$ & $20.0(17.0 ; 21.0)$ & 0.017 \\
\hline & Delta & $-2.0(-6.3 ; 1.0)$ & $0.0(-1.0 ; 0.0)$ & 0.042 \\
\hline \multirow[t]{3}{*}{ PSV RVA [cm/s] } & Supine & $44.0(37.8 ; 46.0)$ & $45.0(43.0 ; 46.0)$ & 0.05 \\
\hline & Sitting/Upright & $37.0(36.0 ; 39.0)$ & $44.0(43.0 ; 46.0)$ & $<0.001$ \\
\hline & Delta & $-4.0(-9.0 ;-1.0)$ & $0.0(-1.0 ; 1.0)$ & $<0.001$ \\
\hline \multirow{3}{*}{ EDV RVA $[\mathrm{cm} / \mathrm{s}]$} & Supine & $12.5(11.0 ; 14.0)$ & $9.0(8.0 ; 11.0)$ & $<0.001$ \\
\hline & Sitting/upright & $9.0(8.0 ; 11.0)$ & $9.0(7.0 ; 10.5)$ & 0.26 \\
\hline & Delta & $-2.5(-5.0 ;-1.0)$ & $0.0(-1.0 ; 0.0)$ & $<0.001$ \\
\hline
\end{tabular}

EDV — end diastolic velocity; PSV - peak systolic velocity; LCCA - left common carotid artery; LICA — left internal carotid artery; LVA — left vertebral artery; RCCA — right common carotid artery; RICA — right internal carotid artery; RVA — right vertebral artery

tions may underestimate the real state of the defect and its clinical effects [13]. Orthostatic-induced changes in Doppler echocardiographic measures of transaortic gradient in patients with AS have been reported recently [14]. Standing is a fundamental activity of daily life and may induce a fall in cardiac patients predisposed to syncope recognized as an important problem in patients with cardiac disease, especially with LV-preload dependence. The orthostatic test is a provocative maneuver that is definitely physiologically based and most relevant to the conditions under which patients predisposed 


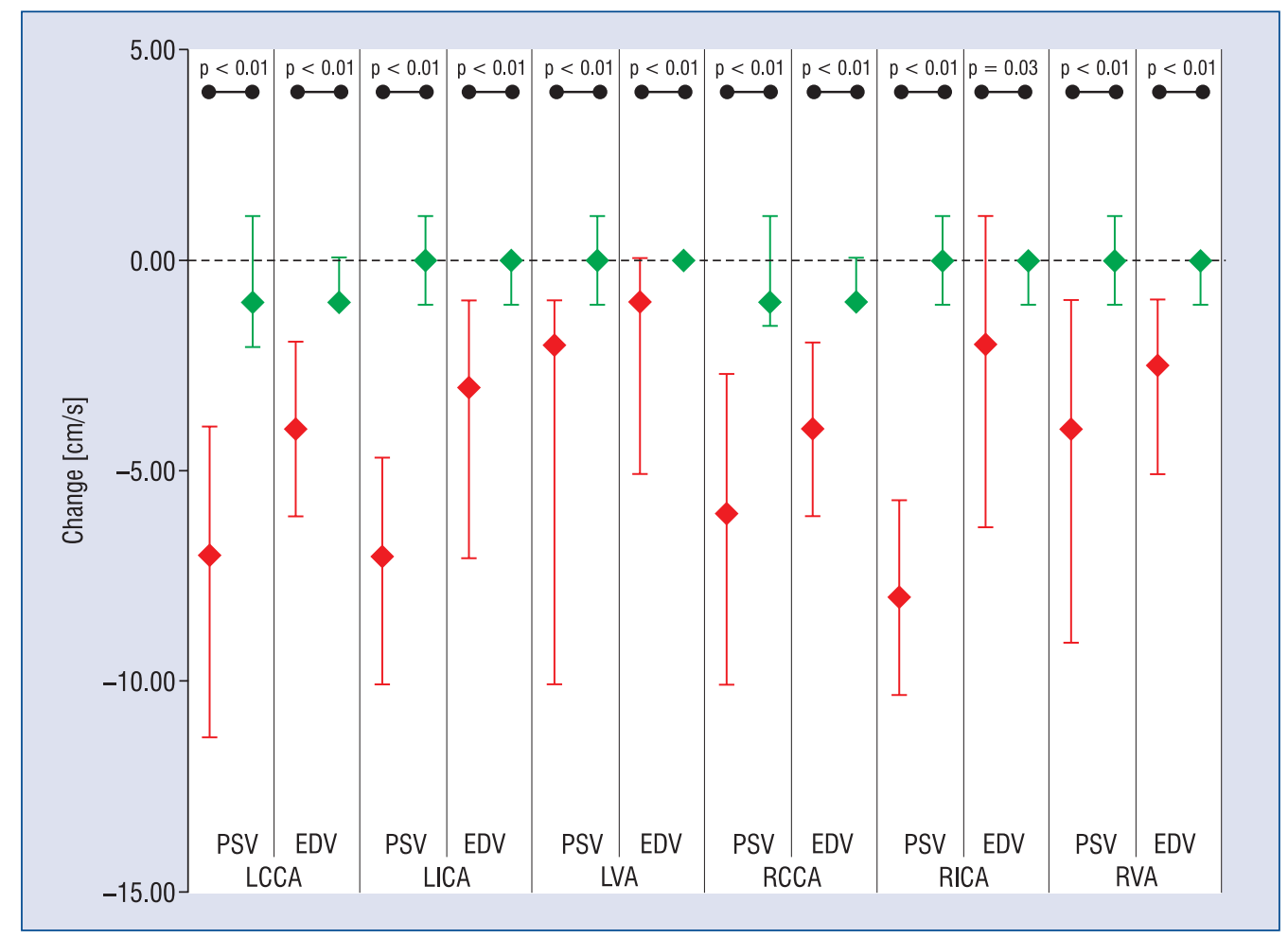

Figure 1. Change in peak systolic velocity (PSV) and end diastolic velocity (EDV) between supine and sitting/upright position for patients with aortic stenosis (red) and healthy controls (green). Data are median with upper and lower quartiles; LCCA — left common carotid artery; LICA — left interial carotid artery; LVA — left vertebral artery; RCCA — right common carotid artery; RICA — right interial carotid artery; RVA — right vertebral artery.

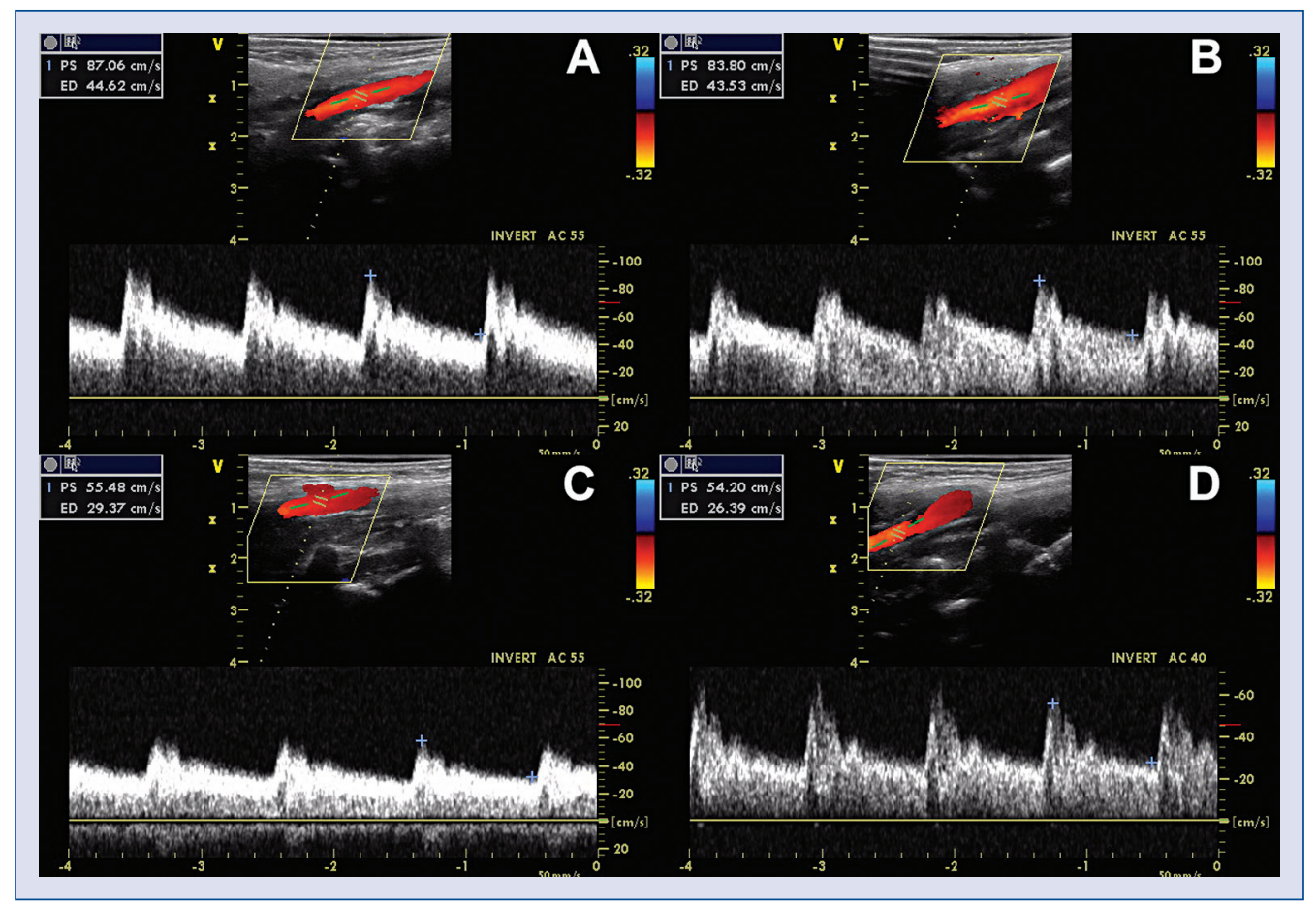

Figure 2. Doppler ultrasound assessment in a patient with severe aortic stenosis (A, B) and a healthy subject (C, D). 
to presyncope present with symptoms. In patients with hypertrophic cardiomyopathy, standing is recommended as a physiologic provocative maneuver [15] and in some patients, stress test as based on standing challenge may guide the therapy. On the other hand, it remains unclear whether orthostatic stress evokes regional differences in cerebral blood flow. Cerebral blood flow in humans is greater when supine compared with when seated or in an upright position [16]. Sato et al. [17] showed that blood flow in the internal carotid artery and medial cerebral artery was reduced during head up tilt test, but vertebral artery blood flow was maintained by dilatation of territories of the vertebrobasilar system. Furthermore, Ogoh et al. [18] have recently provided data that the effect of graded orthostatic stress on vertebral artery blood flow is different from that on internal carotid artery blood flow. This response allows for the possibility that orthostatic tolerance may be associated with hemodynamic changes in posterior rather than anterior cerebral blood flow. In the current study, we observed a statistical trend towards more pronounced drop of blood flow velocity in vertebral arteries $(p=0.15$ for left vertebral arteries and $p=0.11$ for right vertebral arteries) during sitting position, when compared to carotid arteries.

In our study, we sought to investigate the impact of orthostatic stress (unloading LV) on transaortic pressure gradients and carotid and vertebral artery blood-flow waveforms in patients with severe AS. The aortic gradient was examined in upright position and remained concordant with previously published studies $[13,19,20]$. Moreover, there was a significant correlation between transaortic gradient drop during upright position and presyncope. What is more, we found a significant decrease in flow volume after orthostatic stress in patients with AS. Unfortunately, we could not find any correlation between presyncope and blood flow in carotid and vertebral arteries during orthostatic (sitting) stress. Orthostatic stress to assess carotid arteries was performed in a sitting position (similar to previous studies $[20,21]$ ) due to difficulties in carotid Doppler assessment in standing position. This could influence the drop of transvalvular pressure gradient by approximately only $10 \%$, compared to $15 \%$ in other publications [14]. The same may refer to carotid Doppler ultrasound findings and be the reason of insufficient velocity drop that could otherwise eventually correlate with presyncope. A subanalysis with a control group, in which subjects were assessed in standing position, showed a significant blood velocity differences (reduced response) in patients with AS, examined in sitting position. Furthermore, the blood velocity drop could be probably even higher if AS patients were examined also in a standing position. However, still, this is a challenge and probably there is a need for orthostatic testing performed while patient being examined in a lower position than an physician in the echocardiographic laboratory room to obtain a real gradient and velocity drop.

\section{Limitations of the study}

The exclusion criteria of the study significantly constrained patient recruitment and resulted in a relatively small sample size. In the upright position, only the apical window was used for aortic gradient measurement. Another limitation is that examination after orthostatic stress was performed in a sitting position and not standing in patients with AS.

\section{Conclusions}

Lower carotid and vertebral arterial flow velocities in the sitting position do not appear to be associated with a history of syncope/presyncope in patients with severe isolated AS. We found a significant blood velocity drop in carotid and vertebral arteries in patients with AS (sitting position) compared to healthy subjects (standing position). Whether non-invasive estimation of the changes in cerebral blood flow in response to the orthostatic stress test may be helpful to predict the risk of future syncope, requires large prospective studies.

\section{Acknowledgements}

The publication of this paper was supported by the Faculty of Medicine, Jagiellonian University Medical College, Leading National Research Center (KNOW) 2012-2017.

\section{Conflict of interest: None declared}

\section{References}

1. Iung B, Baron G, Butchart EG et al. A prospective survey of patients with valvular heart disease in Europe: The Euro Heart Survey on Valvular Heart Disease. Eur Heart J, 2003; 24: 1231-1243 .

2. Nkomo VT, Gardin JM, Skelton TN et al. Burden of valvular heart diseases: A population-based study. Lancet, 2006; 368: 1005-1011.

3. Joshi S, Patel UK, Yao SS et al. Standing and exercise Doppler echocardiography in obstructive hypertrophic cardiomyopathy: The range of gradients with upright activity. J Am Soc Echocardiogr, 2011; 24: 75-82.

4. Maron MS, Olivotto I, Zenovich AG et al. Hypertrophic cardiomyopathy is predominantly a disease of left ventricular outflow tract obstruction. Circulation, 2006; 114: 2232-2239. 
5. Mason DT, Braunwald E, Ross J. Effects of changes in body position on the severity of obstruction to left ventricular outflow in idiopathic subaortic stenosis. Circulation, 1966; 33: 374-382.

6. Miranda R, Cotrim C, Cardim N et al. Evaluation of left ventricular outflow tract gradient during treadmill exercise and in recovery period in orthostatic position, in patients with hypertrophic cardiomyopathy. Cardiovasc Ultrasound, 2008; 6: 19.

7. Dimitrow PP, Bober M, Michałowska J et al. Left ventricular outflow tract gradient provoked by upright position or exercise in treated patients with hypertrophic cardiomyopathy without obstruction at rest. Echocardiography, 2009; 26: 513-520.

8. Dimitrow PP, Cheng TO. Standing position alone or in combination with exercise as a stress test to provoke left ventricular outflow tract gradient in hypertrophic cardiomyopathy and other conditions. Int J Cardiol, 2010; 143: 219-222.

9. Shah JS, Tome Esteban MT, Thaman R et al. Prevalence of exercise induced left ventricular outflow tract obstruction in symptomatic patients with non-obstructive hypertrophic cardiomyopathy. Heart, 2008; 94: 1288-1294.

10. Cotrim C, Loureiro MJ, Simoes O et al. Evaluation of hypertrophic obstructive cardiomyopathy by exercise stress echocardiography. New methodology. Rev Port Cardiol, 2005; 24: 1319-1327.

11. Cotrim C, Lopes LR, Almeida AR et al. Efficacy of beta-blocker therapy in symptomatic athletes with exercise-induced intraventricular gradients. Cardiovasc Ultrasound, 2010; 8: 38.

12. Dimitrow PP, Michałowska J, Sorysz D. The effect of hemodialysis on left ventricular outflow tract gradient. Echocardiography, 2010; 27: 603-607.

13. Lancellotti P, Rosenhek R, Pibarot P et al. ESC Working Group on Valvular Heart Disease position paper: Heart valve clinics:
Organization, structure, and experiences. Eur Heart J, 2013; 34: 1597-1606.

14. Dimitrow PP, Sorysz D. Orthostatic stress echocardiography as a useful test to measure variability of transvalvular pressure gradients in aortic stenosis. Cardiovasc Ultrasound, 2013; 11: 15.

15. Elliott PM, Anastasakis A, Borger MA et al. Grupa Robocza Europejskiego Towarzystwa Kardiologicznego (ESC) do spraw rozpoznawania i postepowania $\mathrm{w}$ kardiomiopatii przerostowej. 2014 ESC Wytyczne ESC dotyczące rozpoznawania i postępowania w kardiomiopatii przerostowej w 2014 roku. Kardiol Pol, 2014; 72: 1054-1126.

16. Eicke BM, von Schlichting J, Mohr-Ahaly S et al. Lack of association between carotid artery volume blood flow and cardiac output. J Ultrasound Med, 2001; 20: 1293-1298.

17. Sato K, Fisher JP, Seifert T et al. Blood flow in internal carotid and vertebral arteries during orthostatic stress. Exp Physiol, 2012; 97: 1272-1280.

18. Ogoh S, Sato K, Okazaki K et al. Blood flow in internal carotid and vertebral arteries during graded lower body negative pressure in humans. Exp Physiol, 2015; 100: 259-266.

19. Petkow Dimitrow P, Cotrim C, Cheng TO. Need for a standardized protocol for stress echocardiography in provoking subaortic and valvular gradient in various cardiac conditions. Cardiovasc Ultrasound, 2014; 12: 26.

20. Sundstedt M, Hedberg P, Jonason T et al. Doppler assessments of left ventricular filling and ejection during upright exercise in endurance athletes. Clin Physiol Funct Imaging, 2007; 27: 36-41.

21. Rowland T, Unnithan V, Garrard M et al. Sex influence on myocardial function with exercise in adolescents. Am J Hum Biol, 2010; 22: 680-668. 